Trinity University

Digital Commons@ Trinity

Library Faculty Research

Coates Library

2006

\title{
Visual Information Literacy Via Visual Means: Three Heuristics
}

Benjamin R. Harris

Trinity University, bharris@trinity.edu

Follow this and additional works at: https://digitalcommons.trinity.edu/lib_faculty

Part of the Library and Information Science Commons

\section{Repository Citation}

Harris, B.R. (2006). Visual information literacy via visual means: Three heuristics. Reference Services Review, 34(2), 213-221. doi: $10.1108 / 00907320610669452$

This Post-Print is brought to you for free and open access by the Coates Library at Digital Commons @ Trinity. It has been accepted for inclusion in Library Faculty Research by an authorized administrator of Digital Commons@ Trinity. For more information, please contact jcostanz@trinity.edu. 
Visual Information Literacy Via Visual Means:

Three Heuristics

\author{
Benjamin R. Harris \\ Reference/Instruction Librarian \\ Elizabeth Huth Coates Library \\ Trinity University \\ One Trinity Place \\ San Antonio, TX 78212-7200
}

Ph: 2109998160

Fax: 2109998182

E-mail: Benjamin.Harris@ Trinity.Edu 


\section{The Author:}

Benjamin R. Harris holds the position of Reference/Instruction Librarian at Trinity University's Elizabeth Huth Coates Library in San Antonio, Texas, USA.

\section{Category:}

General Review

\section{Abstract:}

Purpose:

To offer definitions and application scenarios for three interdisciplinary heuristics designed to encourage a more holistic view of texts with the objective of raising awareness and enhancing the information literacy of student researchers.

Design/Methodology/Approach:

Based on the thesis that visual texts and images should be considered in information literacy theory and practice, a selection of three visual heuristics found to be useful in instruction session situations are explained and described in a practical teaching situation.

Findings:

These three heuristics can be used in a number of ways for different audiences to encourage critical thinking about the context, components, and the communication process involved in presenting texts used by students (from books, to journal and newspaper articles, and web sites).

Research Limitations/Implications:

There are other useful heuristics that have not been considered within the scope of this study. Other readers and researchers may locate and discuss other means by which these ends can be achieved.

Practical Implications:

An appendix includes a number of scenarios utilizing these heuristics that might be applicable within almost any information literacy session regardless of course topic. However, these heuristics can be applied to course topics if appropriate. Suggestions are also included along with the discussion of each instructional strategy.

Originality/Value:

A number of texts in the professional literature have discussed whether or not visual literacy and images should be considerations for information literacy advocates. Few have offered specific interdisciplinary examples that might be used to experiment with or achieve such an aim. 


\section{Visual Information Literacy Via Visual Means: \\ Three Heuristics}

\section{$\underline{\text { Introduction }}$}

While the terms "information" and "information literacy" are meant to encompass the world of information, images and visually dominant texts are rarely included within their realms. In the academy, alphabetic text remains hegemonic after 500 years of the dominance of print discourse. In the streets, on screens, across our webs, the visual is primary. Icons erase words from desktops, textbooks for all ages have become drenched in images, and the ad-related phrase "sex sells" rarely refers to well ordered paragraphs. Today's information seekers use computers, televisions, and telephones---almost simultaneously---in what seems to be an almost constant swirl of search-find-search again activity.

The reality of the contemporary information economy is that images are in higher circulation and higher demand than words and print. This should not suggest that we create an opposing hierarchy where images rule words, but understanding the shifting relationship between word and image at this juncture in information literacy theory is imperative. While few have questioned the fact that verbal and alphabetic literacies must be learned, a lack of sensitivity to (or fear of) images and visual texts has obscured the need for instruction in reading images. As images become ever-present in the communication of information between entities, communities, and individuals, librarians and related professionals must consider the visual in any discussion of information literacy. 
The topic of information literacy and its kinship with images and visual components of texts has received little attention in the professional literature. Embracing the possibilities of technology and learning, James W. Marcum has written that to separate visual information from traditional alphabetic information is short-sighted when considering the opportunities provided to libraries and their patrons with advancing technology. Traditional print culture, in which images were less easily reproduced, has been decentered in favor of the current visual culture. Marcum advocates that librarians understand and make use of developments in our "visual ecology," contending that "libraries must be transformed into multi-media based services in order to grasp the ephemeral but omnipresent interactivity, to perceive the totality of today's visual ecology, and to manage continuous media that today's culture will not be lost" (Marcum, 201). Such a call to action has direct implications for information literacy advocates, and only three years after Marcum's text appeared, the feasibility of creating digital repositories seems to have given his words greater weight.

More recently, Nerissa Nelson contends that visual literacy instruction is outside of the librarian's instructional focus. According to Nelson, "unlike the concept of information literacy, the concept of visual literacy revolves around the content analysis of images, their meaning, not so much about the technical skills of finding them and their qualitative evaluation in terms of authenticity, currency, etc.” (Nelson, 8). While Nelson's argument includes resources supporting the depth of the visual literacy definition, the stipulative and static way that information literacy is defined within her argument is troublesome. Concepts and theory that attempt to refine information literacy 
to a discrete set of tasks is reductive in light of the changing, advancing, fast-fingered world of information and the media of delivery through which information is traveling.

Further, information literacy is not "mutually exclusive" in relation to the other literacies. Functional reading literacy, print literacy, media literacy, visual literacy, and the new "multimodal" literacies discussed in some disciplines all interact along with information literacy strategies. While information literacy instruction is grounded on certain faculties and an understanding about how information works, it must remain open and adaptable as technologies and their users change and advance.

Still, it is true that few students graduating today will ever be required to submit a visual argument to their instructor, and they may never be asked to find, evaluate, or use images in the course of their studies. The default one inch margined text in 12 point font reigns supreme in student-produced work (for the moment). However, one of the characteristics of information literacy as a concept is that its acquisition will lead to lifelong learning and knowledge formation for individuals receiving instruction. Since most of our students will come in contact with millions of images during the course of their life, and will rarely encounter an 8-page essay written in Microsoft Word with an APA bibliography, lifelong continuous learning is enhanced with a consideration of the multi-textual and highly visual character of information production and presentation. Such re-visions must occur in our professional literature related to the theory and practice of information literacy, and should also occur in our classrooms, our webbed locales and our brick \& mortar spaces.

After speaking on the necessity and value of integrating the visual in information literacy instruction at the 2004 ACRL conference, a number of audience members and 
web attendees asked how to make this happen. The desire was there, but the theory and experience has yet to find its place although it has been a topic of interest and concern in disciplines across academic subjects. Communications scholars, artists and art historians, writing instructors, and others have been examining the developing relationship between word and image (a 100 year old affair spurred by the economical reproduction of images and text that include images). Drawing on the theory of varying disciplines involved in these topics offers routes toward manageable and still effective instructional strategies that recognize the complexities of contemporary texts and multiple literacies required to read them.

\section{The Visual in the Classroom}

It may seem like an inordinate task for information literacy instructors to include discussion or instruction on images within an already swollen battalion of tasks and processes, often condensed into one or two meetings with a class or group of individuals. One cannot expect such a shift to be easy. And yet, as visuals continue to enter the classroom (as well as the written text) at an ever-increasing rate, teachers must find efficient methods for dealing with the influence of the visual.

Interdisciplinary heuristics function as useful tools to assist instructors and students in understanding how information is constructed and received within specific contexts. In addition, these heuristics have been theorized in visual terms, reinforcing the pedagogical benefits of using teaching methods that consider visual learners and learning while also encouraging a deeper understanding of the matrix of relationships that are involved in the textual moment. When faced with the text, readers are rarely dealing with 
alphabetic images only (and after all, words are actually images that represent sound). While previously considered in relation to the construction and transmission of "traditional" informational forms, these heuristics are equally suited to the contextualization and interrogation of visual texts. They encourage consideration of images as information, but they are not specific to visual texts. As opposed to including visual images or visual literacy in information literacy instruction, these heuristics refrain from excluding them.

\section{Vickery's Model of Information Transfer}

A linear heuristic commonly used by instructors in the social sciences (including library studies) for thinking about the communication process is available in the work of B.C. Vickery. Vickery's model of information transfer focuses on the method through which textual messages are transmitted and suggests that there will be a variation in the information event from person to person, even though the information involved in the process is the same. Vickery's formula traces the steps and influences that are involved in the transfer of textual messages (Vickery and Vickery, 42):

Fig. 1: Vickery's Model of Information Transfer

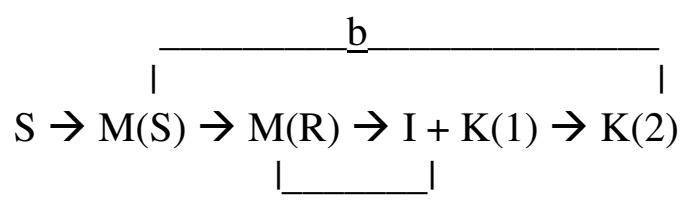

a 
This formulaic diagram includes the following abbreviations to more efficiently depict the process of information transfer: $S=$ source, $M(S)=$ message sent; $\rightarrow=$ channel, $M(R)=$ message received, $I=$ information from message, which changes knowledge state $K(1)$ of individual to $\mathrm{K}(2)$. The information from the message is therefore dependent on the message being received, followed by a change or increase in the receiver's knowledge.

The innovation offered by Vickery's visualization of the information transfer process exists in the upper and lower brackets, loops that can occur in the information and knowledge transmission relationship. The lower feedback loop (a) indicates that the channel may be adjusted to try to change any disparities between the message being sent and the message being received. This means that the encoder can alter the message for a particular audience or recipient. The encoder, in this cause, could be an author or a publisher of information.

The upper loop (b) indicates feedback that has the potential to change the sent message. What environmental influences may influence the relay of information? What outside forces could interrupt the flow of information? Who or what on either the message sender's side of the formula or the message receiver's side influence the message? If a message is verbal or alphabetical, could images or visuals act as interference for the written message? While the process of information transfer seems fairly self explanatory, the questions that may arise due to the upper and lower loops offer numerous instructional opportunities.

Instructors may utilize Vickery's model in discussing how information is transmitted. For example, an article from a scholarly journal available in a full-text database could be analyzed using the formula. Then, the formula can be applied to a 
print version of the text featuring images (either images within or related to the text or advertising images that accompany the print version). A quick review of the differences in the two formulas would offer students suggestions for thinking about (1) receiving information through varying delivery media and (2) how the images included in one medium relate to the readers knowledge in a different way than images provided (or removed) from an alternative medium.

A comparative view using Vickery's model of information transfer would accomplish a number of aims. First, students would think about how information is transmitted and received. Second, students would think about the influence of the authority (both author and information provider) of the information in relation to an eventual audience. And finally, this strategy is a quick way to encourage critical readers to think about how images are involved in their readings. Still, Vickery's critics tend to cite his linear form as being too reductive or simplistic. The following example suggests a less linear fashion of looking at texts and communicative relationships.

\section{The Communication Triangle}

Designed to facilitate discourse study, or "the study of the situational uses of the potential of the language," James L. Kinneavy's communication triangle is a heuristic common to the rhetoric/writing classroom (Kinneavy, 22). Kinneavy's triangle consists of four points that encapsulate the textual relationship: the encoder (or writer), the decoder (or reader), the reality (or the culture, time, and situation of the decoder in relation to the text), and the signal (or text). Kinneavy's construction is not entirely new, since different disciplines had formerly used similar visual devices to try to understand 
the communication situation (Kinneavy, 19).

Fig. 2: Kinneavy's Communication Triangle

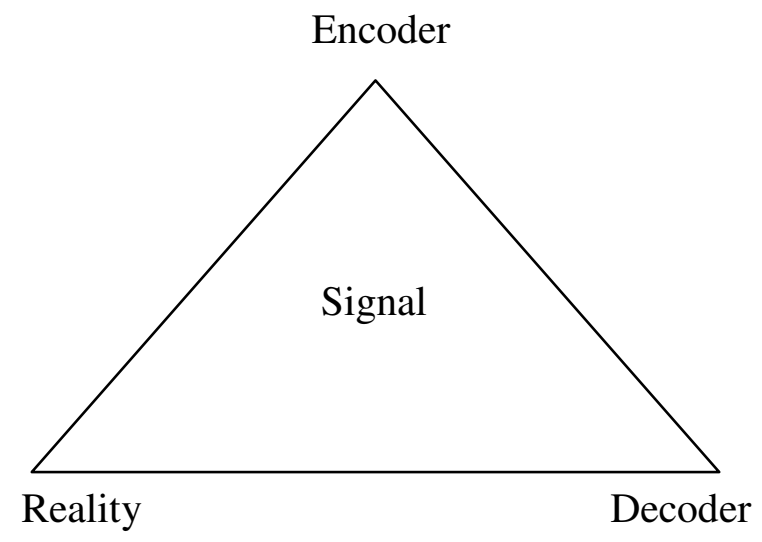

When applied to the visual text, Kinneavy's terms do not require revision. This makes the heuristic particularly useful for interdisciplinary instruction. The terms encoder, decoder, reality, and signal can be defined, clarified, or simplified as far as terminology. The signal selected for analysis could include almost any type of text delivered through almost any type of medium. The use of this heuristic to examine the relationships involved in the textual moment allows the analyst to examine the discursive possibilities of the image beyond a simplistic author/viewer framework, to see how the cultures and rhetorical desires of each are complicit in the construction and reception of the image.

An instructor using the communication triangle may select a text from an in-class search or make use of course texts as examples for showing the way information has been communicated. In Figure 3, a communication triangle has been created for a textbook from a geophysics course. The encoders include the writer and publisher. The decoders 
in this case are a particular class of students. The reality reflects the current situation of the students; however, the same triangle could be constructed with students from 10 years ago as decoders. In this case, the "reality" for those students from a decade past would be somewhat different.

Fig. 3 C.M.R. Fowler; Cambridge UP

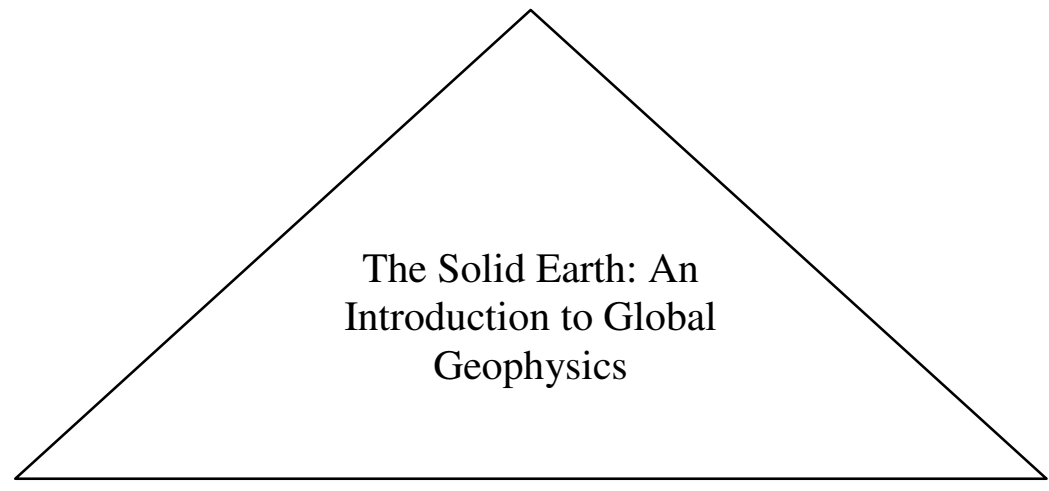

Spring 2004

Geosciences 101

18-24 year old, born mid-80s

University setting

Technologically rich field of study

Computer use common

Etc.

Using this example, questions can be formed to understand the interplay of influence in the communication process. Instead of thinking of the encoder as the originator of information, how does the reality of the time in which it is read effect what the encoder has created? How does this reality affect the way decoders read the text? Could the decoder's perspective on who the encoder is have a direct relationship to how the text is received (as in the case of a canonical work)? And how do images play a role?

Again, instructors seeking to include this model in information literacy sessions have options as well as constraints. The primary benefit of the model is that information 
flows on a continuing current. Editors of the textbook in Fig. 3 may use decoder reception and changes in the reality (time, culture, location, etc.) when creating a new edition of the text. Changes in decoder learning styles due to the reality of the times may require considerations such as more images. Textbooks, as well as other texts, include images in a way that would have been unfamiliar to students 10 years ago—even more so for students from two decades past.

Kinneavy's model requires readers to think critically about the information and how it is presented based on outside influences. It requires readers to consider context in the evaluation of sources, and can be utilized to encourage readers to think about how the relationship between images and alphabetic text is shaped not only by the author or authorial agency, but by the needs and desires of readers. While Vickery requires critical thinking about how different media of delivery can change the information found by the seeker, Kinneavy's triangle requires that readers consider how the time and context in which texts are produced or read relate to the message.

\section{Relational Mapping}

Relational mapping is a common teaching and learning strategy that seeks to depict relationships between things, ideas, or people through visual means. As a more common heuristic, often experienced by students in their elementary grades and up, it can be more easily adapted to new situations and discussions. For thinking about the relationship between images as information and alphabetic text as information, relational maps are useful in a very different manner than either of the prior models. 
Information literacy instructors, and others, are challenged to encourage readers to think about the sources that they use as they locate, select, and use information. For many information seekers, the tunnel-vision that accompanies the work of searching for certain facts, figures, or information components to fulfill a specific need can blind them to the information source itself. One of the benefits of evaluating sources, aside from encouraging critical thinking about source selection, is that it requires the researcher to consider where the information is coming from and how it has been presented.

Relational maps can be as simple or as complicated as the creator desires. Figure 4 shows an example of a fairly simple relational map. In the center of the map is the text under discussion. The components of the text are then broken down and considered separately.

Fig. 4: Relational Map

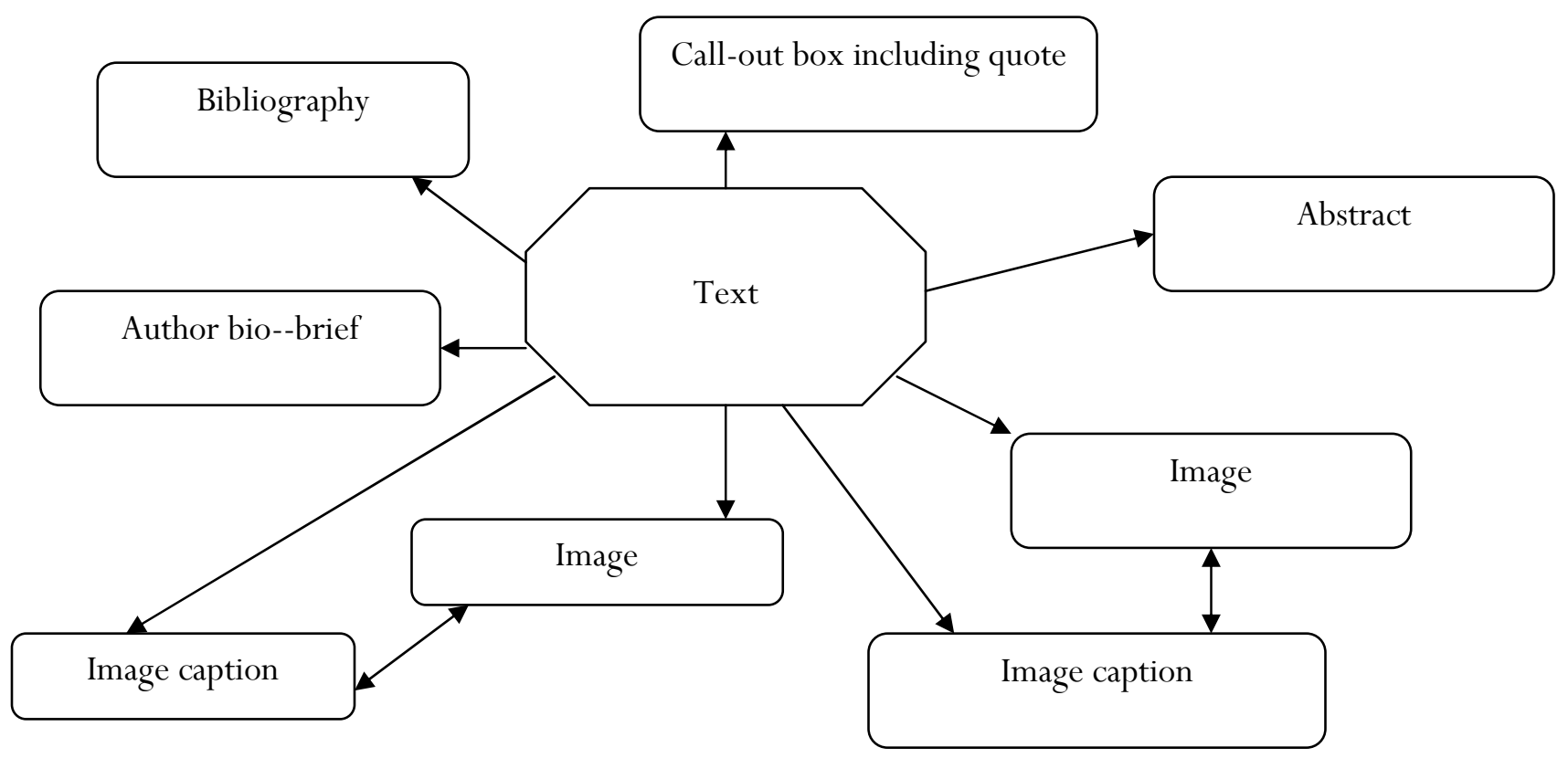


A more complex text may require a more complex map. Ultimately, the goal for the reader is to see all of the different components that join in this textual moment so that they may consider the varying components that are tied to this text. In library studies, such configurations have been utilized by Richard P. Smiraglia to depict "bibliographic families" and by Elaine Svenonius to depict works and "superworks" to explain how varying texts and textual components are related to one another, and how this relationship effects or relates to an originary text. The same principle is involved here, but on a simplified level that focuses on the components of a single source.

For information literacy instructors, this tool asks students to think about all of the parts that make up the text. The text of a critical essay, journal article, or newspaper article rarely exists completely on its own without other articles, advertisements, editorial introductions, et al. In evaluating sources of information, readers are aided by considering how various components shape the information provided, or at the very least, how these components shape their reception of that information. For example, what does a photo of the writer do to the information? What about the author's byline? How is the title valuable or a bibliography, graphs, images, quotations that have been set apart and bracketed, etc.? Such a view of the whole text allows readers to evaluate the source itself and not skew the evaluation to aid in their use of the information found in a tunnel-vision reading.

For teachers, this strategy takes very little time and again, as a familiar visual teaching tool, it can be quickly adapted for this situation. In addition, it requires readers to think about how images and design elements can relate to their evaluations. Will students sit down and construct a relational map for every source they locate? No, and 
this should be qualified within the instruction period. These visual imprints for thinking about sources may not become habitual tools in the lives of researching students, but a greater awareness of the text has been raised.

\section{$\underline{\text { Raising Awareness }}$}

These heuristics offer three ways of looking at texts that offer avenues for the consideration of visual images. Vickery's model focuses on the system of information transmission and medium of delivery; Kinneavy's communication triangle considers context of creation and reception of the text; and relational mapping considers the greater components of the text. It should be reiterated that it may not only be challenging due to time limitations but also difficult in relation to topical requirements in library instruction to offer extended focus on images. However, they must not be excluded, and since they are omnipresent in the texts and tools we introduce in the classroom, to disregard them is exclusion. These strategies allow instructors to broach the subject of how information is created, sent, and received in an inclusive manner that would recognize the multimodal character of contemporary publishing.

It is exactly the work of raising awareness as well as consciousness that makes information literacy a concept that can extend beyond students lives as students. And still, stipulative definitions of information literacy that confine it to a limited series of tasks seem to prevail in some circles. Arguments against considering images as information will continue. Some of these arguments may stem from pedagogical theory that encourages library instructors to attach instruction directly to course content. This is a worthy aim in maintaining student engagement and faculty support of library 
instruction. Consequently, raising awareness about information literacy and a greater comprehension of the information economy is an added value that can be easily constructed into instruction session curricula. While students may not always be information literate, we should not underestimate their information savvy and miss opportunities to enhance their prior knowledge with new considerations. In one-shot teaching and similar situations, raising awareness can often be as valuable as extensive instruction of greater depth.

In addition to serving as methods for introducing how visual images ARE information, these heuristics also enfranchise visual learners who may be set apart from instruction that is exclusively verbal or alphabetic. Introducing these topics through visual methods such as a linear formula and triangular or map-like constructions presents an alternative to verbal lecture or PowerPoint instruction. And every learner is a visual learner in some capacity and in varying degrees. Strategies such as the three discussed here recognize this and enhance continuing work to diversify the library instruction environment.

Further, these strategies may be introduced and discussed at different points in the sequence of a single session or multiple sessions. Making connections between the entities and communicative situations that influence critical readings and use of texts can be connected to explaining the difference between popular and scholarly journals, the impact of the peer review process, the difference between seeing a text in its print or electronic context, as well as other topics commonly introduced in information literacy sessions. (See the appendix for active learning applications of these heuristics.) 
Revising practices for offering standard information literacy instruction sessions are the not the only fronts on which the profession is dealing with the influence of images. For those disciplines that utilize images in the classroom and in student work, teaching- librarians may offer instruction related to image databases such as ARTstor and digital image libraries online. Discussions related to the location, selection, evaluation, and use of images have expanded beyond the arts and communications disciplines to other academic spheres now challenged to integrate images into their curriculum. The influence of the visual will increase. Where this influence will take us, or where we will go with it, remains to be seen. 


\section{Appendix: Integration Strategies}

The following integration strategies for the heuristics discussed in the text have been found to be efficient and effective when introducing these formulas and models in the library instruction classroom. Note that a brief explanation of the heuristic must accompany its use, but that such an explanation should be included only by way of guiding the class. Vickery's model, the communication triangle, and relational mapping are best utilized in an application rather than extended definition.

A. During discussions on the availability of electronic journals and print journals, introduce Vickery's model to explain how reader reception is different when a text is read in print or online. Ask students to consider the points in the flow of information that would be different based on medium. Discuss the impact of an article including images and an article without images to further illustrate this point.

B. Locate and review a newspaper article that includes a captioned photograph, but present the class with only the text on their first viewing. Then show the newspaper article in its original context with the captioned photograph intact. Ask students to consider which points on Vickery's model of information transfer are altered by these two different viewings.

C. In a discussion on the range or depth of a particular text, introduce the communication triangle to explain why texts are geared to certain audiences. Select a text that would be appropriate to course topics and determine if the class is an intended audience for the text by using the triangle. 
D. In discussing the value of publication dating in different disciplines (i.e., science journals focus on more recent evidence as opposed to history journals who would continue to use older sources), use the communication triangle to look at different editions of a work and determine if the most recent edition of that text is necessary. E. Present the class with only the text of a short article (an overhead projector or similar device would be most appropriate for this activity). Slowly add the other components that accompany the information, such as images, titles, author information, etc. while asking students to offer critical thinking on how the article is changed by the added components. Upon completion, quickly construct a relational map that accomplishes the same breakdown of components in less time.

F. Introduce the home page of a website to the class. Ask students to list all of the components seen above the fold on the home page. As students answer, create a relational map that shows the various components. Ask students to evaluate the page and then rank the components based on importance for (1) readers of the site and (2) students using the site as a research source. The use of images on the website's home page may be a focus of inquiry. 


\section{References}

Fowler, C.M.R., (2005) The Solid Earth: An Introduction to Global Geophysics, Cambridge University Press, Cambridge.

Harris, B.R., (2005) “'Big Picture’ Pedagogy: The Convergence of Word and Image Information Literacy Instruction," Currents and Convergence: Navigating the Rivers of Change: Proceedings of the ACRL $12^{\text {th }}$ National Conference, ALA, Chicago.

Kinneavy, J.L., (1971) A Theory of Discourse: The Aims of Discourse, University of Texas Press, Austin.

Marcum, J.W. (2002) "Beyond Visual Culture: The Challenge of Visual Ecology," Libraries and the Academy, 2, 2, 189-2-6.

Nelson, N., (2004) "Visual Literacy and Library Instruction: A Critical Analysis," Education Libraries, 27, 1, 5-10.

Smiraglia, R.P., (2001) The Nature of “A Work": Implications for the Organization of Knowledge, Scarecrow Press, Lanham.

Svenonius, E., (2000) The Intellectual Organization of Information, MIT Press, Cambridge.

Vickery, B.C., and Vickery, A., (1987) Information Science in Theory and Practice. Butterworths, London. 